\section{Avaliação do grau de implantação dos atributos da atenção primária à saúde como indicador da qualidade da assistência prestada às crianças}

\author{
Evaluating the degree of implementation of \\ primary health care attributes as an indicator \\ of quality of care provided to children
}

\section{Evaluación del grado de implantación de los atributos en la atención primaria de salud, como indicador de calidad en la asistencia facilitada a niños}

\section{Resumo}

O objetivo deste artigo foi avaliar o grau de implantação dos atributos da atenção primária à saúde (APS) como indicador da qualidade da assistência prestada às crianças. Realizou-se estudo transversal em um município de médio porte de Minas Gerais, Brasil. Foram entrevistados 707 cuidadores de crianças de 0 a 4 anos (498 da área urbana e 209 da rural) e 22 profissionais das equipes de saúde da família, utilizando o Primary Care Assessment Tool, o critério Brasil de classificação socioeconômica e um questionário elaborado pelos pesquisadores. Foram calculados os escores dos atributos da APS a partir da opinião de usuários e profissionais. Escores $\geq 6,6$ foram considerados indicativos de alto grau de implantação dos atributos. O teste de MannWhitney foi utilizado para comparar os resultados das áreas urbana e rural. Os profissionais avaliaram melhor os serviços da APS do que os usuários. Para os profissionais, os componentes com maiores escores foram "sistemas de informação" e "orientação familiar" (ambos 8,9). Para os usuários, os componentes melhor avaliados foram "sistemas de informação" $(7,8)$ e "utilização" $(6,8)$, e os pior avaliados foram "integração de cuidados" $(4,0)$ e "serviços disponíveis” (4,6). Os escores dos atributos essencial e geral dos serviços da área rural foram mais altos do que os da área urbana na opinião dos usuários, mas não na dos profissionais. Há divergências importantes entre as avaliações de profissionais e usuários. Os serviços da área rural foram melhor avaliados do que os da área urbana, embora os atributos da APS não estejam devidamente implantados no município como um todo, indicando que a qualidade da assistência à criança se encontra aquém da necessária.

Atenção Primária à Saúde; Assistência à Saúde; Qualidade da Assistência à Saúde; Criança; Pesquisa sobre Serviços de Saúde
George Sobrinho Silva 1

Claudia Regina Lindgren Alves 2

doi: 10.1590/0102-311X00095418

Correspondência

G. S. Silva

Rua Sebastião Leopoldo Guedes 82, Diamantina, $M G$ 39100-000, Brasil.

georgesobrinho@yahoo.com.br

1 Universidade Federal dos Vales do Jequitinhonha e Mucuri, Diamantina, Brasil.

2 Universidade Federal de Minas Gerais, Belo Horizonte, Brasil. 


\section{Introdução}

O sistema público de saúde brasileiro tem tido visibilidade internacional por ter alcançado expressivas melhorias nos indicadores de saúde que estão diretamente relacionadas ao desempenho da atenção primária à saúde (APS) ${ }^{1}$. Os maiores destaques foram a redução das taxas de mortalidade infantil e o alcance da meta estabelecida pelos Objetivos do Milênio, pactuados com a Organização das Nações Unidas (ONU) 2. Apesar disso, as taxas de mortalidade infantil no Brasil ainda são maiores que as encontradas em países como Japão, Chile e Argentina, cujos valores são 2,0, 6,6 e 9,8 por mil nascidos vivos, respectivamente $3,4,5,6$. Nos últimos anos, o país voltou a conviver com problemas como o recrudescimento de doenças imunopreveníveis e o aumento das taxas de mortalidade infantil, resultado da grave crise político-econômica que assola o país 7 .

A APS se desenvolve a partir de ações de saúde individuais e coletivas, focadas no diagnóstico e tratamento precoces das condições mais prevalentes, na vigilância em saúde, prevenção de agravos e promoção da saúde, orientados pelos determinantes de saúde das coletividades 8 . O modelo de organização da APS brasileira se baseia, predominantemente, na Estratégia Saúde da Família (ESF), cuja assistência é prestada por equipes multiprofissionais inseridas no ambiente familiar e comunitário 9,10 .

Apesar das evidências de sua efetividade, a APS no Brasil ainda enfrenta dificuldades relacionadas ao seu financiamento, qualificação dos profissionais, insuficiência de gestão, dentre outras 10,11. Para o enfrentamento desses desafios, o Ministério da Saúde vem implementando iniciativas, como a disponibilização do manual do Primary Care Assessment Tool (PCATool-Brasil) 12,13, e a criação de programas como o Programa Nacional para a Melhoria do Acesso e da Qualidade da Atenção Básica (PMAQ-AB), o Programa Mais Médicos (PMM) e o Requalifica UBS 14.

Sendo um dos instrumentos de avaliação da APS propostos pelo Ministério da Saúde, o PCATool avalia o grau de presença e extensão dos atributos "essenciais" (acesso de primeiro contato, longitudinalidade, integralidade e coordenação) e "derivados” (orientação familiar, orientação comunitária e competência cultural) 13. Com a valorização da prática avaliativa, tem crescido o número de estudos utilizando o PCATool 13, propiciando melhor entendimento dos desafios que ainda afetam a APS no Brasil 15,16.

Entendendo a importância de superar as limitações da APS para a retomada do processo de melhoria das condições de vida e saúde da população, torna-se necessário compreender quais são os aspectos macroestruturais, contextuais e organizacionais que determinam a capacidade de resposta dos serviços de saúde às demandas da sociedade. O desenvolvimento de estudos avaliativos é uma das ferramentas disponíveis para lidar com esse cenário. O presente estudo tem por objetivo avaliar a qualidade da assistência à saúde da criança, a partir da análise do grau de presença e extensão dos atributos da APS, conforme proposto pelo PCATool-Brasil, no Município de Diamantina, Minas Gerais.

\section{Métodos}

Trata-se de um estudo transversal realizado nas áreas urbana e rural de Diamantina, município localizado no Alto Jequitinhonha, Minas Gerais. Diamantina possui uma população estimada em 48.095 habitantes (Instituto Brasileiro de Geografia e Estatística. http://www.ibge.gov.br, acessado em 06/ Fev/2017), e sua APS dispõe de 14 equipes de saúde da família (EqSF), sendo oito na área urbana, quatro em áreas rurais e duas unidades mistas, conferindo cobertura de cerca de $74 \%$ da população urbana e 100\% da população rural (Sistema de Informações da Atenção Básica. http://www2.datasus. gov.br/SIAB/index.php?area=04, acessado em 06/Fev/2017).

Foram entrevistados cuidadores (pais ou parentes próximos considerados responsáveis pelo cuidado) de crianças de 0 a 4 anos de idade, que fossem cadastradas na unidade básica de saúde (UBS) de sua área de abrangência e que tivessem utilizado esses serviços, pelo menos, uma vez nos últimos seis meses. Foram excluídos cuidadores portadores de distúrbios mentais ou emocionais que impedissem a compreensão dos procedimentos da pesquisa. O Sistema de Informações da Atenção Básica (SIAB) foi utilizado como referência para o levantamento da população adscrita às UBS, de modo que se adotou a mesma faixa etária desse sistema para o cálculo amostral. 
O cálculo amostral para os usuários foi realizado considerando que: $80 \%$ das 3.173 crianças de 0 a 4 anos do município utilizavam os serviços oferecidos ( 2.538 cadastradas nas EqSF), e a proporção de crianças da área urbana para as da área rural era de 2:1, de acordo com dados da Secretaria Municipal de Saúde de Diamantina (Sistema de Informações da Atenção Básica. http://www2.datasus.gov.br/ SIAB/index.php?area=04, acessado em 06/Fev/2017) e do IBGE (Instituto Brasileiro de Geografia e Estatística. http://www.ibge.gov.br, acessado em 06/Fev/2017); poder estatístico de $80 \%$ e nível de $95 \%$ de confiança. A amostra foi estimada em 716 crianças (507 na área urbana e 209 na rural), já acrescidos os $10 \%$ de perdas.

Com base nos cadastros das EqSF, foram obtidos o quantitativo e a distribuição das crianças por cada equipe, considerando a área de residência (urbana ou rural). Os entrevistados foram escolhidos por sorteio aleatório simples, realizado a partir de uma lista prévia de todas as crianças na faixa etária indicada residentes na área de abrangência de cada EqSF. Nos casos em que foram sorteadas mais de uma criança da mesma família, o responsável foi convidado a responder às perguntas em relação à criança mais velha. Nesse caso, outra criança da mesma área foi sorteada e incluída. O mesmo foi feito caso alguma das crianças sorteadas não preenchesse os critérios de inclusão do estudo. Assim, foram sorteadas tantas crianças quantas fossem necessárias para atingir a amostra calculada. Foram entrevistados 707 cuidadores (498 na área urbana e 209 na rural). Foram excluídas nove crianças da amostra urbana, pois, apesar de cadastradas nos serviços de saúde, essas não eram usuárias deles. Não foi possível substituí-las, pois não foram encontradas outras crianças, da mesma área de abrangência, que preenchessem os critérios de inclusão.

Foram elegíveis para a entrevista os médicos e enfermeiros que estivessem trabalhando nas EqSF do município por, pelo menos, 12 meses, o que permitiria o conhecimento da realidade e da assistência prestada pela equipe. Foram excluídos os profissionais que estivessem afastados do trabalho por mais de um mês, por qualquer motivo, durante o período de coleta de dados naquela unidade. Do total de 28 profissionais alocados, 22 foram entrevistados. A coleta de dados coincidiu com o período de novas contratações advindas de concurso público, realizado pela Secretaria Municipal de Saúde, de modo que seis profissionais estavam trabalhando há menos de um ano nas respectivas equipes e foram excluídos da pesquisa.

Antes da coleta de dados, foi realizado estudo piloto em que foram entrevistados seis usuários da área urbana e seis da rural. Os participantes do estudo piloto não foram incluídos na amostra da pesquisa. Não foram necessários ajustes nos instrumentos propostos.

A coleta dos dados foi realizada entre outubro de 2014 e abril de 2015. A partir da lista de crianças sorteadas, os cuidadores foram recrutados durante as visitas aos serviços de saúde ou em seus domicílios. As informações sobre a família e a criança, a condição socioeconômica das famílias e a percepção delas sobre os serviços de saúde foram coletadas por meio de um questionário estruturado elaborado pelos pesquisadores, do Critério Brasil da Associação Brasileira de Empresas de Pesquisa (ABEP. http://www.abep.org/criterio-brasil) e do PCATool-Brasil (versões criança e profissional) ${ }^{12}$, respectivamente. Quando o respondente não era a própria mãe, solicitou-se que o entrevistado fornecesse os dados relativos à mãe da criança sorteada. Cada entrevista durou, em média, cerca de 40 minutos.

Os escores dos atributos essenciais e derivados da APS foram calculados conforme preconizado no manual do PCATool-Brasil. Foram obtidas as médias aritméticas da pontuação atribuída aos itens que compõem cada atributo e seus componentes. Esse valor foi transformado para uma escala de $0 \mathrm{a}$ 10 (Escore APS = [(escore obtido-1)] x 10/3) para o cálculo dos escores. A partir desses, foram calculados o Escore essencial (média dos escores dos atributos acesso de primeiro contato, longitudinalidade, integralidade e coordenação da atenção) e o Escore geral (média de todos os atributos). Os escores com valores maiores ou iguais a 6,6 foram considerados indicativos de forte orientação para os atributos da APS 12. As famílias foram consideradas afiliadas à EqSF quando o nome do profissional e/ ou serviço público de saúde do bairro foram citados em, pelo menos, uma das três perguntas iniciais do PCATool (A1-A3). Quando o nome do profissional e/ou serviço público de saúde do bairro não foi citado espontaneamente, o entrevistador solicitou que o cuidador continuasse a entrevista avaliando o serviço de saúde utilizado pela criança pela última vez 12 .

Os dados foram armazenados numa planilha do software Excel (https://products.office.com/), com dupla digitação. A análise estatística foi realizada no software R, versão 3.3 (http://www.r-pro ject.org). Para a caracterização dos usuários e profissionais, foram calculadas frequências absolutas 
e relativas para as variáveis qualitativas, e medidas de posição, tendência central e dispersão, para as variáveis quantitativas. O teste de Mann-Whitney foi utilizado para comparar os resultados dos seguimentos urbano e rural.

O projeto foi aprovado pelo gestor de saúde do município e pelo Comitê de Ética em Pesquisa da Universidade Federal de Minas Gerais (CAAE: 36519614.0.0000.5149). Todos os participantes assinaram o termo de consentimento livre e esclarecido antes do início das entrevistas. Foram respeitados todos os preceitos éticos estabelecidos pela Resolução no 466/2012 do Conselho Nacional de Saúde.

\section{Resultados}

A Tabela 1 apresenta a descrição das características dos profissionais. Cerca de $60 \%$ dos profissionais atuavam na área urbana, sendo que 63,6\% eram médicos, e 36,4\%, enfermeiros. Dentre os médicos, 35,7\% eram do PMM, sendo que 80\% estavam alocados nas equipes da área rural. Os profissionais eram formados, em geral, em universidades públicas (45,5\%) e trabalhavam na EqSF, em média, há 22,8 meses. A maioria possuía pós-graduação (90,9\%), sendo 40,9\% relacionada à área de Saúde Pública e Saúde da Família (residência e especialização). Entre os profissionais com pós-graduação relacionada à área de Saúde Pública e/ou Saúde da Família, 55,6\% eram os médicos do PMM.

O perfil dos cuidadores, apresentado na Tabela 2, mostra que as mães foram as principais respondentes das entrevistas (82,7\%). Elas foram também consideradas as principais responsáveis pelos

\section{Tabela 1}

Características dos profissionais da atenção primária à saúde de Diamantina, Minas Gerais, Brasil, 2017.

\begin{tabular}{|c|c|c|}
\hline Variáveis & $\mathbf{n}$ & $\%$ \\
\hline \multicolumn{3}{|l|}{ Tipo de unidade de saúde } \\
\hline Urbana & 13 & 59,1 \\
\hline Rural & 5 & 22,7 \\
\hline Mista & 4 & 18,2 \\
\hline \multicolumn{3}{|l|}{ Sexo } \\
\hline Masculino & 11 & 50,0 \\
\hline Feminino & 11 & 50,0 \\
\hline \multicolumn{3}{|l|}{ Formação } \\
\hline Médico & 14 & 63,6 \\
\hline Enfermeiro & 8 & 36,4 \\
\hline \multicolumn{3}{|l|}{ Universidade de formação } \\
\hline Estrangeira & 5 & 22,7 \\
\hline Privada & 7 & 31,8 \\
\hline Pública & 10 & 45,5 \\
\hline \multicolumn{3}{|l|}{ Possui pós-graduação } \\
\hline Sim & 20 & 90,9 \\
\hline Não & 2 & 9,1 \\
\hline \multicolumn{3}{|c|}{ Pós-graduação na área de Saúde Pública/Saúde da Família (residência e/ou especialização) } \\
\hline Sim & 9 & 40,9 \\
\hline \multirow[t]{2}{*}{ Não } & 13 & 59,1 \\
\hline & Média & DP \\
\hline Idade (anos) & 38,9 & 9,5 \\
\hline Tempo de formação (anos) & 10,3 & 5,9 \\
\hline Tempo de trabalho na equipe de saúde da família (meses) & 22,8 & 26,0 \\
\hline
\end{tabular}

DP: desvio padrão. 


\section{Tabela 2}

Características dos usuários responsáveis por crianças de 0 a 4 anos cadastradas nos serviços da atenção primária à saúde de Diamantina, Minas Gerais, Brasil, 2017.

\begin{tabular}{|c|c|c|c|c|}
\hline Variáveis & $\mathbf{n}$ & $\%$ & & \\
\hline \multicolumn{5}{|l|}{ Entrevistados } \\
\hline Mãe & 585 & 82,7 & & \\
\hline Avós & 67 & 9,5 & & \\
\hline Outros & 55 & 7,8 & & \\
\hline \multicolumn{5}{|l|}{ Idade da mãe (anos) } \\
\hline$<19$ & 37 & 5,2 & & \\
\hline$\geq 19$ & 668 & 94,8 & & \\
\hline \multicolumn{5}{|l|}{ Número de filhos } \\
\hline Primíparas & 289 & 40,9 & & \\
\hline Multíparas & 418 & 59,1 & & \\
\hline \multicolumn{5}{|l|}{ Bolsa Família * } \\
\hline Sim & 306 & 43,3 & & \\
\hline Não & 401 & 56,7 & & \\
\hline \multicolumn{5}{|l|}{ Classificação socioeconômica ** } \\
\hline$A, B$ & 87 & 12,3 & & \\
\hline$C, D, E$ & 620 & 87,7 & & \\
\hline \multicolumn{5}{|l|}{ Serviço de afiliação } \\
\hline ESF & 502 & 74,0 & & \\
\hline \multirow[t]{2}{*}{ Outros serviços } & 176 & 26,0 & & \\
\hline & Média & DP & Mínimo & Máximo \\
\hline Escolaridade da mãe (anos) & 9,4 & 3,3 & 0,0 & 22,0 \\
\hline Vezes que a criança utilizou os serviços de APS nos últimos seis meses & 3,7 & 2,2 & 1,0 & 20,0 \\
\hline Idade da criança (meses) & 29,9 & 17,5 & 0,5 & 64,2 \\
\hline
\end{tabular}

APS: atenção primária à saúde; DP: desvio padrão; ESF: Estratégia Saúde da Família.

* Programa do Governo Federal destinado à transferência de benefício financeiro a famílias em situação de pobreza e extrema pobreza 31;

** Associação Brasileira de Empresas de Pesquisa (ABEP; http://www.abep.org/criterio-brasil).

cuidados com as crianças $(87,8 \%)$, e 5,2\% das mães eram adolescentes. As famílias pertenciam, principalmente, às classes C, D e E (87,7\%), e 43,3\% recebiam o benefício Bolsa Família. Embora todos os entrevistados fossem usuários dos serviços de APS de Diamantina, apenas 74\% dos cuidadores informaram espontaneamente o nome do profissional e/ou serviço público de saúde do bairro. Essa proporção foi de $88 \%$ e 63,9\% nas áreas rural e urbana, respectivamente. Nesses casos, o último serviço de saúde utilizado pela criança foi, em geral, a UBS do próprio bairro.

A Figura 1 apresenta a análise descritiva dos escores atribuídos por profissionais e usuários para cada atributo da APS. A linha tracejada representa o escore 6,6. Escores acima desse ponto de corte sugerem forte implantação dos atributos ou seus componentes. Para os profissionais, a APS apresentou forte grau de implantação para a maioria dos atributos avaliados, com destaque para os componentes "sistemas de informações" $(9,0)$ e "orientação familiar" $(8,9)$. Dentre os atributos que não atingiram o escore mínimo de qualidade, o componente "acessibilidade" foi o que obteve a pior avaliação (4,2). O Escore essencial teve média de 6,7 \pm 1,2, enquanto o Escore geral médio foi de 6,9 $\pm 1,1$.

$\mathrm{Na}$ avaliação dos usuários, os atributos da APS estão, em geral, com escores abaixo do ponto de corte de 6,6. O componente "sistema de informações" $(7,8)$ e "utilização" $(6,8)$ apresentaram os maiores escores médios. Já "integração de cuidados" $(4,0)$ e "serviços disponíveis" $(4,6)$ tiveram as menores médias. O Escore essencial teve média igual a 6,0 $\pm 1,7$, e o Escore geral apresentou média de 5,8 \pm 1,7, conforme apresentado na Figura 1. 
Figura 1

Escores dos atributos da atenção primária à saúde (PCATool) na avaliação de profissionais e usuários. Diamantina, Minas Gerais, Brasil, 2017.

1a) Profissionais

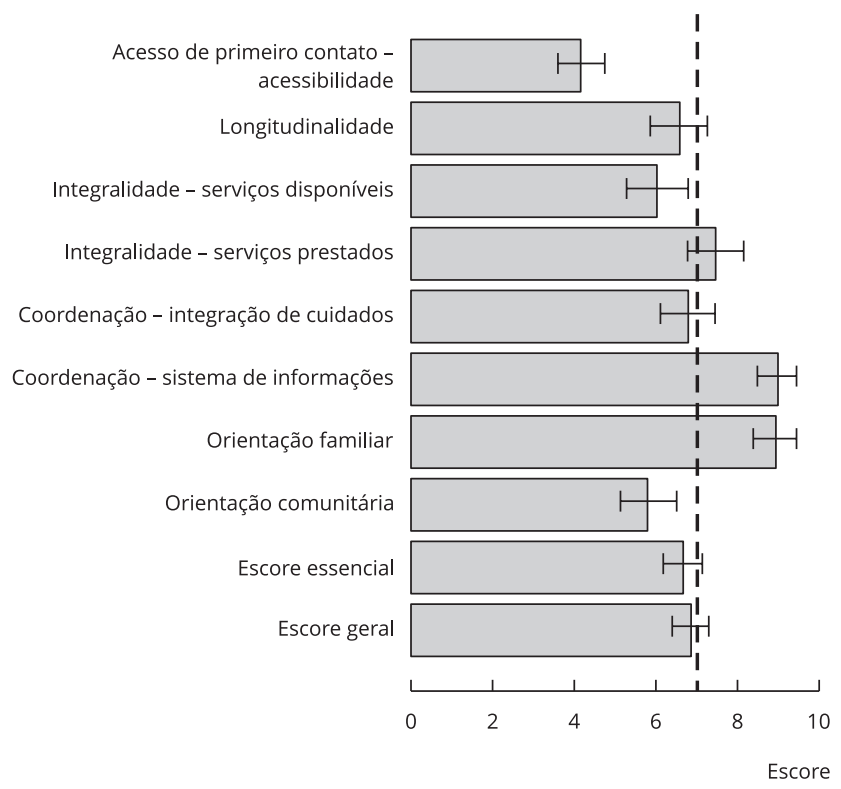

1b) Usuários

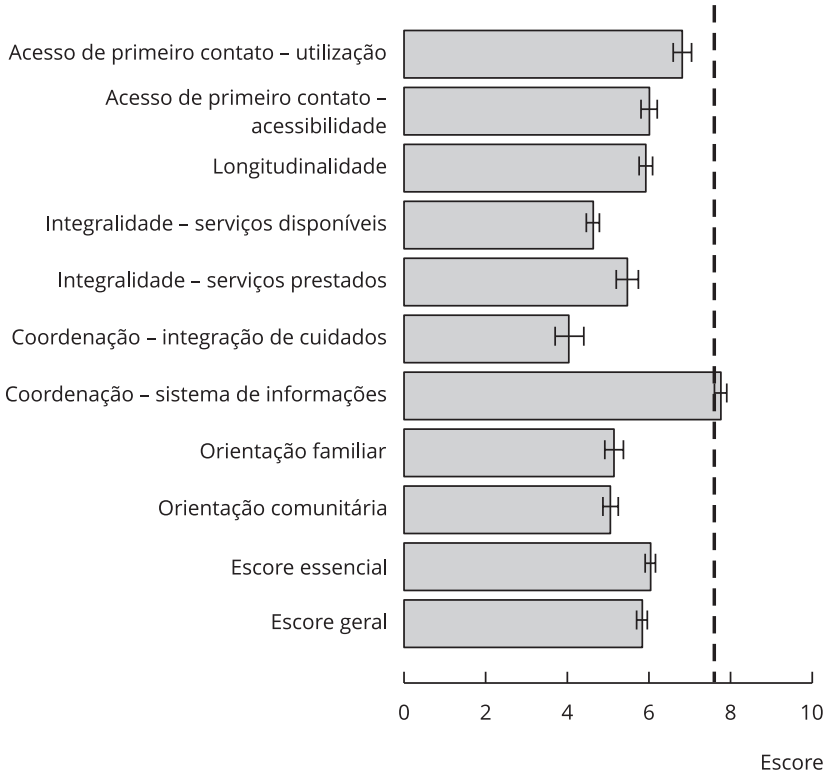

A análise comparativa do desempenho dos serviços nas áreas urbana e rural na opinião de profissionais e usuários é apresentada na Tabela 3. Na opinião dos profissionais, apenas o atributo "acessibilidade” mostrou-se significativamente maior na área rural do que na área urbana $(\mathrm{p}=0,042)$. Já na opinião dos usuários, os componentes "utilização" ( $<<0,001)$, "acessibilidade” ( $<<0,001)$, "longitudinalidade" ( $<<0,001)$, "integração de cuidados" ( $p<0,001)$, "sistema de informações” $(p<0,001)$ e "orientação familiar" ( $p=0,007)$ apresentaram escores médios significativamente superiores na área rural. Na opinião dos usuários, os escores essencial e geral também foram significativamente mais altos na área rural. Não houve diferença significativa entre as áreas urbana e rural em relação aos demais atributos.

A Figura 2 ilustra os resultados apresentados na Tabela 3, evidenciando as diferenças nas avaliações de usuários e profissionais sobre as áreas urbana e rural.

\section{Discussão}

O perfil dos profissionais entrevistados coincide com o encontrado em outros estudos 17,18 e mostram que, tradicionalmente, os profissionais das EqSF são jovens e com pós-graduação. Poucos profissionais possuíam qualificação específica para a atuação na APS, o que representa importante obstáculo para a qualidade da oferta dos serviços de saúde 18. Os profissionais com qualificação específica eram, em sua maioria, médicos do PMM e estavam concentrados nas EqSF da área rural. É plausível pensar que a qualificação específica dos profissionais possa ter contribuído para a melhor avaliação da assistência nas EqSF da área rural. Por outro lado, o processo de transição na forma de contratação dos profissionais de saúde no município durante o período de coleta de dados acarretou a exclusão de quase metade dos enfermeiros, fato que também pode ter interferido com a percepção dos usuários, pois ainda não teriam tido tempo de se vincular aos novos profissionais. 


\section{Figura 2}

Comparação do desempenho dos serviços da atenção primária à saúde das áreas urbana e rural, de acordo com a avaliação de profissionais e usuários em Diamantina, Minas Gerais, Brasil, 2017.

2a) Profissionais

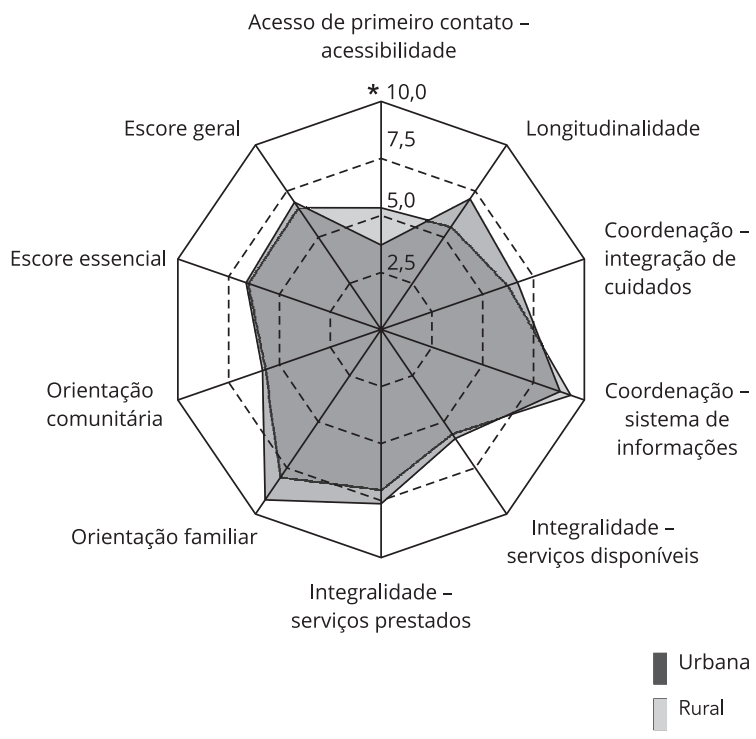

2b) Usuários

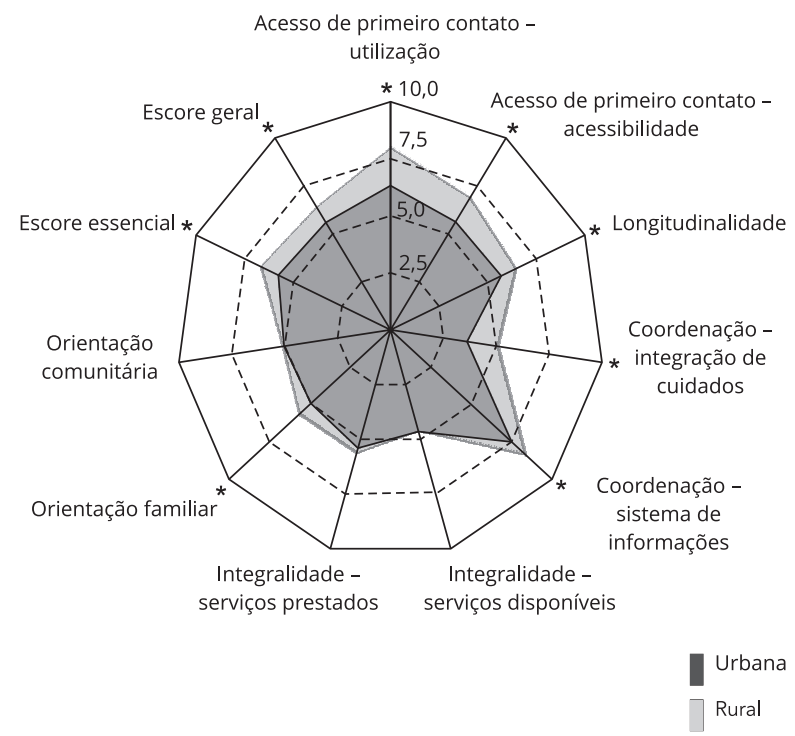

\section{Tabela 3}

Comparação da avaliação feita por profissionais e usuários quanto ao desempenho dos serviços da atenção primária à saúde das áreas urbana e rural de Diamantina, Minas Gerais, Brasil, 2017.

\begin{tabular}{|c|c|c|c|c|c|c|c|c|c|c|}
\hline \multirow[t]{3}{*}{ Atributos } & \multicolumn{5}{|c|}{ Profissionais ( $N=22$ ) } & \multicolumn{5}{|c|}{ Usuários (N = 707) } \\
\hline & \multicolumn{2}{|c|}{ Urbana $(n=13)$} & \multicolumn{2}{|c|}{ Rural (n=9) } & \multirow[t]{2}{*}{ Valor de $p$ * } & \multicolumn{2}{|c|}{ Urbana $(n=498)$} & \multicolumn{2}{|c|}{ Rural (n = 209) } & \multirow[t]{2}{*}{ Valor de $p$ * } \\
\hline & Média & EP & Média & EP & & Média & EP & Média & EP & \\
\hline $\begin{array}{l}\text { Acesso de primeiro contato - } \\
\text { utilização }\end{array}$ & - & - & - & - & - & 6,3 & 0,1 & 7,9 & 0,2 & 0,000 \\
\hline $\begin{array}{l}\text { Acesso de primeiro contato - } \\
\text { acessibilidade }\end{array}$ & 3,7 & 0,3 & 5,3 & 0,6 & 0,042 & 5,6 & 0,1 & 6,9 & 0,2 & 0,000 \\
\hline Longitudinalidade & 7,1 & 0,5 & 5,6 & 0,7 & 0,093 & 5,7 & 0,1 & 6,5 & 0,1 & 0,000 \\
\hline $\begin{array}{l}\text { Coordenação - integração de } \\
\text { cuidados }\end{array}$ & 6,7 & 0,5 & 6,2 & 0,4 & 0,343 & 3,6 & 0,2 & 5,1 & 0,35 & 0,000 \\
\hline $\begin{array}{l}\text { Coordenação - sistema de } \\
\text { informações }\end{array}$ & 8,8 & 0,4 & 9,3 & 0,4 & 0,490 & 7,5 & 0,1 & 8,4 & 0,1 & 0,000 \\
\hline $\begin{array}{l}\text { Integralidade - serviços } \\
\text { disponíveis }\end{array}$ & 5,9 & 0,6 & 5,6 & 0,6 & 0,961 & 4,7 & 0,1 & 4,6 & 0,1 & 0,867 \\
\hline $\begin{array}{l}\text { Integralidade - serviços } \\
\text { prestados }\end{array}$ & 7,6 & 0,5 & 7,0 & 0,6 & 0,458 & 5,4 & 0,2 & 5,6 & 0,3 & 0,431 \\
\hline Orientação familiar & 9,2 & 0,3 & 8,0 & 0,8 & 0,266 & 4,9 & 0,2 & 5,7 & 0,2 & 0,007 \\
\hline Orientação comunitária & 5,8 & 0,4 & 5,7 & 0,8 & 0,790 & 5,0 & 0,1 & 5,1 & 0,2 & 0,777 \\
\hline Escore essencial & 6,6 & 0,4 & 6,5 & 0,4 & 1,000 & 5,8 & 0,1 & 6,7 & 0,1 & 0,000 \\
\hline Escore geral & 6,9 & 0,3 & 6,6 & 0,4 & 0,767 & 5,6 & 0,1 & 6,4 & 0,1 & 0,000 \\
\hline
\end{tabular}

EP: erro padrão.

* Teste de Mann-Whitney. 
O perfil dos usuários entrevistados também se assemelha ao encontrado em outros estudos 16,19 que se propuseram a avaliar a assistência à criança na APS. A amostra foi composta principalmente pelas mães das crianças, apontadas como as principais responsáveis pelo cuidado de seus filhos. As famílias, em sua maioria, eram de baixa renda e muitas eram beneficiárias de programas de distribuição de renda. Esses achados reiteram o papel da mulher como principal responsável pelo lar nas famílias brasileiras, sobretudo naquelas de menor poder aquisitivo 16.

Os dados mostram que a EqSF é apontada como a principal fonte de assistência à saúde das crianças, semelhante ao encontrado em outros estudos realizados nas cidades de Pouso Alegre 16 e Alfenas 19, em Minas Gerais. Outros estudos 17,20 apontam também que o modelo assistencial baseado em EqSF tem maior capacidade de responder às demandas de saúde da população, sobretudo para as crianças, do que os modelos tradicionais. Apesar disso, um quarto dos entrevistados não citou espontaneamente o nome do profissional e/ou serviço de saúde da EqSF de seu bairro, sugerindo um vínculo fraco com o serviço de referência para o cuidado da criança, como pode ser observado na avaliação dos atributos.

Foram observadas importantes divergências na percepção de usuários e profissionais, o que é comumente encontrado em estudos avaliativos que envolvem múltiplos atores 17,21,22. Esse fato pode estar relacionado à diferença do nível de conhecimento dos atores sobre os itens em questão, à existência de conflitos entre eles, à divergência de interesses etc. 17,21,22. Estudos 17,23,24 que avaliaram a APS com a mesma metodologia apontam que profissionais tendem a avaliar melhor a qualidade dos serviços. Já os usuários e cuidadores tendem a ser mais críticos 16,17. Serapioni \& da Silva 22 afirmaram que a qualidade dos serviços de saúde não pode ser definida apenas pela avaliação de um único ator, seja ele gestor, profissional ou usuário, sob o risco de reduzir a abrangência da avaliação. De acordo com Castro et al. 17, a maioria dos estudos avaliativos privilegiam a opinião dos usuários, por serem aqueles que, de fato, têm a experiência de uso dos serviços e cuja satisfação é um parâmetro norteador na busca da qualidade na assistência em saúde. No entanto, essa abordagem isolada é considerada insuficiente para determinar a qualidade dos serviços 22 .

Outro contraste identificado neste estudo refere-se às avaliações dos usuários de serviços nas áreas urbana e rural, indicando divergências na percepção do grau de implantação dos atributos nos diferentes segmentos. Acredita-se que essa diferença esteja relacionada ao perfil de oferta e utilização dos serviços em cada área, que são condicionados pelas diferenças entre as populações e pelos aspectos organizacionais das EqSF 25,26, além das diferenças no perfil dos profissionais atuando em cada seguimento.

Embora se norteiem pelos mesmos princípios organizativos estabelecidos pela Política Nacional da Atenção Básica 5, a funcionalidade das EqSF urbana e rural se dá de forma distinta. A área rural de Diamantina é composta por vilas e comunidades, dispersas em grandes extensões territoriais. Isso determina a necessidade de deslocamento contínuo dos profissionais, que prestam assistência de forma itinerante, diferentemente do que acontece nas unidades da área urbana. Apesar de o modelo de funcionamento das equipes que cobrem a área rural parecer insuficiente por não ser contínuo, deve-se ressaltar que essas comunidades distantes ficaram totalmente desassistidas por muitos anos, de modo que esse atendimento, mesmo que intermitente, mas constante e previsível, pode gerar satisfação nos usuários. Pode-se considerar também que os profissionais que atuam na área rural possuam habilidades específicas que proporcionem maior vínculo apesar do contato não tão frequente com os usuários.

Sobre os atributos da APS, a "atenção de primeiro contato" foi avaliada nos componentes "utilização" e "acessibilidade”. O componente "utilização", que é avaliado apenas pelos usuários, aborda o elemento processual do acesso à APS, que se refere ao primeiro serviço a ser procurado pela família, em caso de necessidade para um novo problema de saúde 27 . Os dados mostraram que esse atributo está fortemente implantado na área rural, e fracamente na área urbana, onde não atingiu o escore de qualidade. $\mathrm{O}$ menor escore do atributo na área urbana pode estar relacionado à possibilidade de os usuários recorrerem a outras fontes de cuidado para a criança. Diferentemente, nas áreas rurais, a APS é a opção mais provável, visto que a possibilidade de acesso a outros serviços é mais remota.

O baixo escore do componente "utilização" sinaliza a existência de obstáculos para o acesso das crianças aos serviços, apesar de outros estudos 16,20,28 apontarem que a EqSF é preferida para assistência das crianças pela melhor qualidade do atendimento e vínculo com os profissionais. Dentre os 
principais obstáculos para o acesso e a utilização dos serviços, foram citadas as barreiras geográficas e a pressão da demanda por ações curativas, advinda da grande prevalência de eventos agudos na faixa pediátrica, levando à sobrecarga dos serviços 19 .

O componente "acessibilidade" trata da estrutura disponível para o acesso aos serviços e se refere a horários, dias, localização, disponibilidade, comodidade e conveniência dos serviços 27 . O escore desse componente ficou abaixo do índice de qualidade, tanto na percepção dos usuários quanto na dos profissionais, sendo pior avaliado entre os profissionais. Nesse atributo, as maiores fragilidades relacionam-se à indisponibilidade dos serviços em fins de semana e no horário noturno e de um telefone de referência para os usuários no período em que as unidades estão fechadas. O horário de funcionamento das EqSF na área urbana pode não atender às expectativas dos usuários, muitos com normas mais rígidas no ambiente do trabalho e que dificultam o acesso aos serviços de saúde 21. A maior disponibilidade de outros tipos de serviços, como consultórios privados e unidades de pronto atendimento, pode também aguçar o senso crítico dos usuários em relação à acessibilidade e a outros atributos da APS urbana 20,21,29. O elevado tempo de espera dos usuários para receber atendimento também contribuiu para o baixo desempenho nesse atributo na opinião dos profissionais, refletindo a capacidade de organização das equipes e a demanda por ações imediatas 21,30 .

O atributo da "longitudinalidade" também não alcançou o escore de qualidade na perspectiva de usuários e profissionais. Na avaliação dos usuários, as áreas rurais alcançaram o escore de qualidade, diferentemente da urbana. A longitudinalidade se refere à existência de uma conexão entre usuários e prestadores, em que ambos reconhecem o compromisso pelo cuidado, o que pressupõe uma relação intensa que expressa fidelidade e confiança ${ }^{27}$. A avaliação recebida nesse atributo sugere a dificuldade na criação do vínculo entre serviço e usuários, a falta de interação com a família e com a história de vida da criança 31. A melhor avaliação desse atributo pode acontecer quando há o esforço dos profissionais em assistir e resolver as necessidades dos usuários. A menor vinculação dos usuários da área urbana aos serviços de APS pode estar relacionada à maior facilidade de acessar outros recursos para a assistência à saúde, como especialistas e mesmo outros recursos de apoio diagnóstico, sem a devida coordenação das EqSF 16.

O atributo da "integralidade" está ligado à capacidade da APS reconhecer as necessidades de saúde da população adscrita e disponibilizar um leque de serviços para atender essas necessidades. Esse atributo também foi avaliado em dois componentes, "serviços disponíveis", que se refere à estrutura e à variedade de ações disponíveis na APS, e "serviços prestados", que versa sobre a disponibilidade de ações de prevenção e promoção à saúde. O componente "serviços disponíveis" não alcançou o índice de qualidade nem na avaliação dos profissionais nem dos usuários. O desempenho desse atributo é apontado como consequência da insuficiente qualificação dos profissionais e do pouco conhecimento deles sobre as reais necessidades dos usuários, ou seja, a falta de um diagnóstico adequado da situação de saúde da área de abrangência, sendo acrescentados a falha na organização das redes de atenção para apoio diagnóstico ou terapêutico, o sistema de transporte sanitário e o agendamento de consultas em outros serviços e internações 16.

O componente "serviços prestados" foi bem avaliado pelos profissionais e mal avaliado pelos usuários. Os usuários dos serviços de saúde no Brasil ainda possuem uma concepção de utilização de serviços de saúde arraigada ao modelo biomédico, centrado no consumo de procedimentos médicos, em detrimento das ações de prevenção e promoção 22. A mudança dessa lógica de pensamento é um dos desafios para APS. Embora as políticas de saúde no Brasil orientem que as ações da APS se norteiem pela lógica das ações de prevenção de agravos e promoção à saúde, o que se vê é a persistência de práticas individualistas de procedimentos médicos e distantes da realidade dos usuários 32 . Esse contexto é o reflexo da formação de profissionais ainda centrada na prática clínica e da falta de capacitação específica deles para atuar na APS 18,30.

O atributo "coordenação" corresponde à harmonização entre a APS e a rede de atenção no município. Seu primeiro componente é a "integração dos cuidados", que trata da capacidade de a APS se comunicar com outros serviços de referência, de modo a dar continuidade à assistência aos usuários 27. Observa-se divergência entre as avaliações de profissionais e usuários, novamente com melhor avaliação pelos profissionais e usuários da área rural. A melhor avaliação desse atributo está relacionada à capacidade dos serviços de dar seguimento à assistência iniciada 13 . A não continuidade pode ser o resultado de uma assistência fragmentada, da não responsabilização dos serviços e da 
falha na formação da rede de serviços 16,31. Na área urbana, a possibilidade de os usuários acessarem atendimentos especializados sem a necessidade de encaminhamentos prévios fragiliza o papel das EqSF na integração dos cuidados aos usuários sob sua responsabilidade sanitária e gera insatisfação em profissionais e usuários.

O componente "sistema de informações" fala da disponibilidade de informações sobre a saúde dos usuários e o acesso a elas 24. Esse componente foi o melhor avaliado pelos profissionais e pelos usuários, de ambos os seguimentos, apesar de os sistemas apresentarem limitações, como a não informatização, a baixa qualidade das informações registradas, a não utilização das informações para o planejamento da assistência e as dificuldades na comunicação interprofissional 33,34. O mesmo resultado foi encontrado em outros estudos 17,31. A disponibilidade de prontuários eletrônicos e sistemas integrados possibilitaria o compartilhamento e o acesso oportuno às informações, melhorando a continuidade da assistência e a integração da rede de cuidados 35. É possível que os entrevistados tenham tido alguma dificuldade na avaliação desse componente, indicando alguma fragilidade do PCATool para mensurar a qualidade dos sistemas de informação na APS.

O atributo "orientação familiar" remete à consideração do ambiente familiar pela EqSF como cenário para o cuidado. Esse atributo atingiu o escore de qualidade na avaliação dos profissionais, mas não na dos usuários. Para os usuários, houve diferença significativa entre as áreas rural e urbana, com menor escore na área urbana. A divergência da avaliação pode estar relacionada a diferenças de concepção e de entendimento sobre o atributo. A "orientação familiar" deveria representar a essência do modelo de organização da EqSF, cuja proposta é a assistência centrada na família e no contexto em que ela está inserida ${ }^{17}$. O mesmo pode ser dito para o atributo da "orientação comunitária”, que também não recebeu o escore mínimo de qualidade, de nenhum dos entrevistados e em ambos os seguimentos. Estudos com resultados semelhantes 20,31 sugerem que o desconhecimento das reais necessidades dos usuários e o pequeno número de profissionais com formação específica para atuar na saúde da família podem estar relacionados ao fraco desempenho desses atributos.

Os resultados apresentados refletem o descompasso entre a percepção de profissionais e usuários e sugerem uma avaliação melhor pelos usuários das áreas rurais. A assistência à saúde da criança na APS de Diamantina, de modo geral, apresenta ainda muitas fragilidades quanto ao grau de implantação dos atributos da APS analisados. Esse resultado corrobora com outros encontrados na literatura 20,23,31, e aponta que, apesar das melhorias dos indicadores da saúde da criança nos últimos anos, a qualidade da assistência oferecida à população infantil ainda está aquém da necessária. Por outro lado, os melhores escores atribuídos, em geral pelos usuários residentes na área rural, podem representar algum movimento na direção de se garantir equidade, tendo em vista a maior vulnerabilidade dessas comunidades.

O presente estudo possui limitações inerentes aos estudos transversais, que restringem a generalização dos resultados. Essas limitações podem ter sido minimizadas pelo tamanho da amostra, pela aleatoriedade e estratificação na escolha dos entrevistados. Apesar do uso de instrumento de avaliação validado na literatura internacional, percebe-se que alguns atributos não se ajustam adequadamente ao modelo de APS adotado no Brasil, como é o caso do componente "acessibilidade", ou são de difícil avaliação pelos usuários como o componente "sistema de informação”. Outras abordagens metodológicas poderão contribuir para a compreensão, em profundidade, das divergências entre a percepção de usuários e profissionais e entre as comunidades urbanas e rurais. A percepção dos gestores e a análise da evolução dos indicadores de saúde da criança no município podem também ajudar a preencher as lacunas surgidas a partir do presente estudo.

\section{Conclusão}

Tomando como referência o grau de implantação dos atributos da APS, conclui-se que a assistência à saúde da criança em Diamantina ainda não atingiu plenamente os padrões de qualidade estabelecidos pelo PCATool. Paradoxalmente, foram atribuídos melhores escores entre usuários da área rural quando comparados aos da área urbana em muitos dos atributos estruturantes da APS. Também não houve consenso entre as percepções de usuários e profissionais, estes últimos tendendo a atribuir mellhores escores do que os primeiros. 
Esses resultados refletem a complexidade das questões envolvidas na qualificação da APS no Brasil, perpassando a formação dos profissionais, a fixação deles nas EqSF, a precarização das relações de trabalho, as diferenças nas expectativas de profissionais e usuários, a organização do processo de trabalho e a valorização de ações curativas em detrimento de ações de promoção da saúde e prevenção de agravos, além de questões relacionadas ao financiamento e à gestão dos serviços de saúde. $\mathrm{O}$ caso de Diamantina é capaz de ilustrar perfeitamente a realidade vivida por inúmeros municípios de pequeno-médio porte de nosso país, que veem suas conquistas sociais sendo ameaçadas por questões político-econômicas.

Assim como no restante do país, a expansão da cobertura da APS representa, sem dúvida, um avanço social e o atendimento ao direito essencial à saúde, especialmente para as crianças. No entanto, o expressivo percentual de cuidadores cadastrados pelas EqSF que não se sentem vinculados a elas expõe uma fragilidade importante no sistema vigente. Pode ser atribuída sim a deficiências na formação e preparação dos profissionais para o exercício da profissão nesse modelo assitencial, mas é inevitável falar da alta rotatividade observada nas equipes de Diamantina e na maioria das cidades, atribuída, sobretudo, à precarização das relações de trabalho.

Por outro lado, as divergências de percepção entre cuidadores das áreas urbana e rural revelam não apenas diferentes formas de organização das equipes, mas, principalmente, apontam para diferentes perfis de usuários e de profissionais. A ideia de que a EqSF "vai até onde o povo está" traz o sentimento de reconhecimento dos usuários dos cantões do município enquanto sujeitos de direitos e fortalece os conceitos de acessibilidade e equidade, mas também transparece algum grau de resignação com o pouco que se tem. Já na área urbana, vimos cuidadores mais críticos e, de certa forma, insatisfeitos com o modelo implantado, que talvez não atenda suas expectativas arraigadas nas antigas práticas biologicistas e médico-centradas.

Ao propor avaliar a qualidade da assistência à saúde da criança nos serviços de APS utilizando um instrumento validado no país e reconhecido internacionalmente, nos defrontamos com os desafios de avaliar um sistema complexo e multideterminado. Ouvir usuários e profissionais trouxe à tona questões que merecem desenhos investigativos mais amplos e com maior profundidade, que considerem aspectos macroestruturais, culturais, contextuais e epidemiológicos, e que ajudem a apontar soluções transformadoras do status quo.

\section{Colaboradores}

G. S. Silva participou da concepção do projeto, da coleta e análise dos dados e da redação do manuscrito. C. R. L. Alves participou da concepção do projeto, da análise dos dados e da redação do manuscrito.

\section{Informações adicionais}

ORCID: George Sobrinho Silva (0000-0001-99656576); Claudia Regina Lindgren Alves (0000-00020885-1729).

\section{Agradecimentos}

Coordenação de Aperfeiçoamento de Pessoal de Nível Superior (Capes).

\section{Referências}

1. Johnson CD, Noyes J, Haines A, Thomas K, Stockport C, Ribas AN, et al. Learning from the Brazilian community health worker model in North Wales. Global Health 2013; 9:25.

2. Pedro MV, Rodrigues DF, Silva D. Saúde é o que interessa? A influência do PSF no alcance da meta de redução da mortalidade infantil dos Objetivos de Desenvolvimento do Milênio (2000-2011). Revista Política Hoje 2017; 25:175-212.

3. Moreira LMC, Alves CRL, Belisário SA, Bueno MC. Políticas públicas voltadas para a redução da mortalidade infantil: uma história de desafios. Rev Méd Minas Gerais 2012; 22 Suppl 7:48-55

4. Sousa JS, Campos RT, Silva AF, Bezerra FN, Lira JS. Estimação e análise dos fatores determinantes da redução da taxa de mortalidade infantil no Brasil. Revista Brasileira de Estudos Regionais e Urbanos 2016; 10:140-55. 
5. França E, Lansky S. Mortalidade infantil neonatal no Brasil: situação, tendências e perspectivas. In: Rede Interagencial de Informações para Saúde, organizador. Demografia e saúde: contribuição para análise de situação e tendências. Brasília: Organização Pan-Americana da Saúde; 2009. p. 83-112. (Série G. Estatística e Informação em Saúde). (Série Informe de Situação e Tendências).

6. Central Intelligence Agency. The world factbook. Field listing: age structure. https://www. cia.gov/library/publications/the-world-factbook/rankorder/2091rank.html (acessado em 06/Set/2018).

7. Rasella D, Basu S, Hone T, Paes-Sousa R, Ocké-Reis CO, Millett C. Child morbidity and mortality associated with alternative policy responses to the economic crisis in Brazil: a nationwide microsimulation study. PLoS Med 2018; 15:e1002570.

8. Ministério da Saúde. Política Nacional da Atenção Básica. Brasília: Ministério da Saúde; 2012. (Série E. Legislação em Saúde).

9. Ministério da Saúde. Portaria no 2.436, de 21 de setembro de 2017. Aprova a Política Nacional de Atenção Básica, estabelecendo a revisão de diretrizes para a organização da Atenção Básica, no âmbito do Sistema Único de Saúde (SUS). Diário Oficial da União 2017; 22 set.

10. Paim J, Travassos C, Almeida C, Bahia L, Macinko J. The Brazilian health system: history, advances, and challenges. Lancet 2011; 377:1778-97.

11. Mendonça CS, Diercks MS, Kopittke L. O fortalecimento da Atenção Primária à Saúde nos municípios da Região Metropolitana de Porto Alegre, Brasil, após a inserção no Programa Mais Médicos: uma comparação intermunicipal. Ciênc Saúde Coletiva 2016; 21:2871-8.

12. Secretaria de Atenção em Saúde, Ministério da Saúde. Manual do instrumento de avaliação da atenção primária à saúde: Primary Care Assessment Tool. Brasília: Ministério da Saúde; 2010.

13. Fracolli LA, Gomes MF, Nabão FR, Santos MS, Cappellini VK, Almeida AC. Primary health care assessment tools: a literature review and metasynthesis. Ciênc Saúde Coletiva 2014; 19:4851-60.

14. Pinto LF, Giovanella L. Do Programa à Estratégia Saúde da Família: expansão do acesso e redução das internações por condições sensíveis à atenção básica (ICSAB). Ciênc Saúde Coletiva 2018; 23:1903-14.

15. Malouin R, Starfield B, Sepulveda M. Evaluating the tools used to assess the medical home. Manag Care 2009; 18:44-8.

16. Mesquita Filho M, Luz BS, Araújo CS. A Atenção Primária à Saúde e seus atributos: a situação das crianças menores de dois anos segundo suas cuidadoras. Ciênc Saúde Coletiva 2014; 19:2033-46.
17. Castro RCL, Knauth DR, Harzheim E, Hauser L, Duncan BB. Avaliação da qualidade da atenção primária pelos profissionais de saúde: comparação entre diferentes tipos de serviços. Cad Saúde Pública 2012; 28:1772-84.

18. de Oliveira CS, Fonseca AD, Souza LP, das Graças Siqueira L, Barbosa DA. Qualificação de enfermeiros em saúde da família e qualidade da atenção na ótica de usuários. Ciênc Cuid Saúde 2015; 14:1251-8.

19. da Silva SA, Fracolli LA. Avaliação da assistência à criança na Estratégia de Saúde da Família. Rev Bras Enferm 2016; 69:47-53.

20. Harzheim E, Hauser L, Pinto LF, Soranz D. Avaliação dos usuários crianças e adultos quanto ao grau de orientação para Atenção primária à saúde na cidade do Rio de Janeiro, Brasil. Ciênc Saúde Coletiva 2016; 21:1399408.

21. Reis RS, Coimbra LC, da Silva AA, dos Santos AM, Lamy ZC, Ribeiro SV, et al. Acesso e utilização dos serviços na Estratégia Saúde da Família na perspectiva dos gestores, profissionais e usuários. Ciênc Saúde Coletiva 2013; 18:3321-31.

22. Serapioni M, da Silva MG. Avaliação da qualidade do Programa Saúde da Família em Municípios do Ceará. Uma abordagem multidimensional. Ciênc Saúde Coletiva 2011; 16:431526.

23. da Silva SA, Baitelo TC, Fracolli LA. Avaliação da atenção primária à saúde: a visão de usuários e profissionais sobre a estratégia de saúde da família. Rev Latinoam Enferm 2015; 23:979-87.

24. da Silva SA, Nogueira DA, da Silva Paraizo CM, Fracolli LA. Avaliação da atenção primária à saúde: visão dos profissionais de saúde. Rev Esc Enferm USP 2014; 48(Esp):126-32.

25. Kassouf AL. Acesso aos serviços de saúde nas áreas urbana e rural do Brasil. Revista de Economia e Sociologia Rural 2005; 43:29-44.

26. Arruda NM, Maia AG, Alves LC. Desigualdade no acesso à saúde entre as áreas urbanas e rurais do Brasil: uma decomposição de fatores entre 1998 a 2008. Cad Saúde Pública 2018 34:e00213816.

27. Starfield B, organizador. Atenção primária: equilíbrio entre necessidades de saúde, serviços e tecnologia. Brasília: Organização das Nações Unidas para a Educação, a Ciência e a Cultura/Ministério da Saúde; 2002.

28. Ribeiro LCD, Rocha RL, Ramos-Jorge ML. Acolhimento às crianças na atenção primária à saúde: um estudo sobre a postura dos profissionais das equipes de saúde da família. Cad Saúde Pública 2010; 26:2316-22.

29. Elias PE, Ferreira CW, Alves MC, Cohn A, Kishima V, Escrivão Jr. A, et al. Atenção básica em saúde: comparação entre PSF e UBS por estrato de exclusão social no Município de São Paulo. Ciênc Saúde Coletiva 2006; 11:633-41. 
30. de Oliveira VB, Veríssimo MD. Assistência à saúde da criança segundo suas famílias: comparação entre modelos de atenção primária. Rev Esc Enferm USP 2015; 49:30-6.

31. Marques AS, Freitas DA, Alves Leão CD, Oliveira M, Ketllin S, Pereira MM, et al. Atenção primária e saúde materno-infantil: a percepção de cuidadores em uma comunidade rural quilombola. Ciênc Saúde Coletiva 2014; 19:36571.

32. Costa GD, Cotta RM, Reis JR, Ferreira MD, Reis RS, Franceschini SD. Avaliação da atenção à saúde da criança no contexto da Saúde da Família no Município de Teixeiras, Minas Gerais (MG, Brasil). Ciênc Saúde Coletiva 2011; $16: 3229-40$.
33. Carreno I, Moreschi C, Marina B, Hendges DJ, Rempel C, Oliveira MM. Análise da utilização das informações do Sistema de Informação de Atenção Básica (SIAB): uma revisão integrativa. Ciênc Saúde Coletiva 2015; 20:947-56.

34. Heidemann ITSB, Costa MFBNA, Hermida PMV, Marçal CCB, Cypriano CC. Sistema de informação da atenção básica: potencialidades para a promoção da saúde. Acta Paul Enferm 2015; 28:152-9.

35. Almeida PF, Giovanella L, Mendonça MH, Escorel S. Desafios à coordenação dos cuidados em saúde: estratégias de integração entre níveis assistenciais em grandes centros urbanos. Cad Saúde Pública 2010; 26:286-98. 


\section{Abstract}

This article sought to evaluate the degree of implementation of primary health care (PHC) attributes as an indicator of the quality of care provided to children. We carried out a cross-sectional study in a middle-sized city in Minas Gerais State, Brazil. We interviewed 707 childcare workers who work with children aged 0 to 4 years (498 from the urban area and 209 from the rural area) and 22 professionals from the family health teams, using the Primary Care Assessment Tool, the Brazil criteria for socioeconomic classification and a questionnaire designed by the researchers. We calculated the PHC attribute scores based on opinions from users and professionals. Scores $\geq 6.6$ were considered indicative of a high degree of attribute implementation. We used the Mann-Whitney test to compare results from the urban and rural areas. Professionals rated PHC services more highly than users. To professionals, the components with the highest scores were "information systems" and "family guidance" (both 8.9). To users, the components with the highest scores were "information systems" (7.8) and "use" (6.8) and those with the lowest scores were "care integration" (4.0) and "available services" (4.6). The scores of essential and general service attributes were higher in the rural area than in the urban area according to users, but not according to professionals. There are important divergences between evaluations from professionals and users. Rural-area services were better evaluated than those in the urban areas, despite the fact that PHC attributes have not been adequately implemented in the entire city, indicating that the quality of the care provided to children falls short of what is needed.

Primary Health Care; Delivery of Health Care; Quality of Health Care; Child; Health Services Research

\section{Resumen}

El objetivo de este artículo fue evaluar el grado de implantación de los atributos en la atención primaria de salud (APS), como indicador de la calidad en la asistencia prestada a niños. Se realizó un estudio transversal en un municipio de tamaño medio de Minas Gerais, Brasil. Se entrevistaron a 707 cuidadores de niños de 0 a 4 años (498 del área urbana y 209 de la rural) y 22 profesionales de los equipos de salud de la familia, utilizando el Primary Care Assessment Tool, el criterio Brasil de clasificación socioeconómica y un cuestionario elaborado por los investigadores. Se calcularon las puntuaciones de los atributos de la APS a partir de la opinión de usuarios y profesionales. Las puntuaciones $\geq 6,6$ se consideraron indicativas de un alto grado de implantación de los atributos. El test de Mann-Whitney se utilizó para comparar los resultados de las áreas urbanas y rurales. Los profesionales evaluaron mejor los servicios de la APS que los usuarios. Para los profesionales, los componentes con mayores puntuaciones fueron "sistemas de información" $y$ "orientación familiar" (ambos 8,9). Para los usuarios, los componentes mejor evaluados fueron "sistemas de información" $(7,8)$ y "utilización" $(6,8), y$ los peor evaluados fueron "integración de cuidados" $(4,0)$ y "servicios disponibles" $(4,6)$. Las puntuaciones de los atributos esenciales y generales de los servicios del área rural fueron más altos que los del área urbana, en opinión de los usuarios, pero no según la de los profesionales. Existen divergencias importantes entre las evaluaciones de profesionales y usuarios. Los servicios del área rural se evaluaron mejor que los del área urbana, pese a que los atributos de la APS no estén debidamente implantados en el municipio como un todo, indicando que la calidad de la asistencia al niño se encuentra muy lejana respecto a lo que es necesario.

Atención Primaria de Salud; Prestación de Atención de Salud; Calidad de la Atención de Salud; Niño; Investigación en Servicios de Salud

Recebido em 19/Mai/2018

Versão final reapresentada em 13/Set/2018

Aprovado em 18/Out/2018 